\title{
A Model-Based Coordinated Control Concept for Steam Power Plants
}

\author{
Ali Chaibakhsh, ${ }^{1}$ and Ali Ghaffari ${ }^{2}$ \\ ${ }^{1}$ Department of Mechanical Engineering, University of Guilan, P.O. Box 41635-3756, Rasht, Guilan 41996-13769, Iran \\ ${ }^{2}$ Department of Mechanical Engineering, K.N. Toosi University of Technology, P.O. Box 19395-1999, Tehran 19991-43344, Iran
}

Correspondence should be addressed to Ali Chaibakhsh; chaibakhsh@guilan.ac.ir

Received 25 August 2012; Revised 27 November 2012; Accepted 12 December 2012

Academic Editor: WaiKeung Wong

Copyright (C) 2013 A. Chaibakhsh and A. Ghaffari. This is an open access article distributed under the Creative Commons Attribution License, which permits unrestricted use, distribution, and reproduction in any medium, provided the original work is properly cited.

\begin{abstract}
An application of model-based coordinated control concept for improving the performance and maneuverability of once-through power plants is presented. In this structure, neuro-fuzzy-based Hammerstein models are employed as the core of feedforward (FF) controller to generate the reference trajectories for the plant's subsystems. The setpoints for fuel, electrical power, and steam pressure are provided by FF controller with respect to load demands. In order to diminish the effect of disturbances and to compensate deviations between variables and corresponding feedforward setpoints, feedback controllers are considered. The error signal defined by the deviation of desired boiler output pressure from its actual value is used to compensate the demand signals for turbine and boiler. The performance of the proposed control system is compared with that of a conventional turbine-follower and also turbinefollower coordinated control modes during load changes. The results indicate the effectiveness and load tracking capability of the proposed control system at different operating conditions.
\end{abstract}

\section{Introduction}

Current requirements on safe and economic power generation with higher level of efficiency cause that modernization of aged thermal power plants becomes extremely in consideration. Emerging new technologies in computer science has attained a great attention in employing model-based strategies in process control system such that different classes of computer control algorithms have been developed in this regard $[1,2]$.

The aging effects on thermal power plants could decline the availability and performance of overall system, where increasing the sensitivity and nonlinearities of plant makes the control system design much difficult [3]. Minimizing the boiler and turbine losses during transients, reducing thermal stress, and attaining smooth and stable control actions are the other requirements that have to be attained in order to increase the maneuverability and the economic operation of power plant [4].

The high interacted structure, nonlinear behavior, and exposing to great continuous disturbances will cause that the feedback control could not sustain the plant stability and performance for wide-range operation. In this case, a combination of feedback and feedforward should be used to achieve entire objectives. The successful implementations of feedback-feedforward strategy for wide-range load control of fossil fuel power plant have been reported $[5,6]$.

Developing a nonlinear controller directly based on the system nonlinear model is a technique used to deal with nonlinearity of boiler-turbine, which is generally applied by feedback linearization approaches $[7,8]$. One of the difficulties of such methods is that a nonlinear mathematical model of plant is always required to be developed, where irrefutably a trial and error procedure should be preformed to define its parameters with respect to boundaries, inputs, and outputs $[9,10]$.

Robust techniques are usually better alternatives in order to compensate the system dynamic variations [6]. However, only limited benefits would be obtained by these techniques, and a proper degree of flexibility and maneuverability cannot be easily achieved. A main concern in this regard is that 
the feedforward controller cannot be designed independently from the feedback-loop spectral characteristics $[8,11]$.

There are many other control methods such as adaptive control or gain-scheduling control that are used to deal with the nonlinearities of a power plant. Adaptive techniques are generally required to update the control parameters in sequences of time, which can make them rather complex. However, the changes of dynamic behaviors are very slow, and it is not necessary for the control parameters to be identified frequently. In this scene, gain-scheduling controllers have a greater capability $[12,13]$. A major drawback of this approach is the selection of the scheduling procedure changing the control gains with respect to scheduling variables. Defining control laws is normally preformed in an offline process and for different operating conditions. In this case, unexpected changes in the dynamics of plant may lead to deterioration of performance or even to total failure [14]. As a result, the robustness of controller is an essential requirement, which should be considered to provide safe control of system at different operating conditions [15]. It is shown that the fuzzy supervisory can provide stable and bump less switching under gain scheduling to ensure the dynamic performance of system [16].

Employing the concept of inverse dynamic model in order to derive feedforward action could significantly improve the performance of a control system. This can be adopted by learning the behavior of system based on measured or known desired output trajectories [17]. This concept is employed in new generation of coordinated control system to generate reference trajectories for subsystems. The unit load control, at the uppermost hierarchical level, is responsible for driving setpoints for main control loops of power and steam pressure to match the boiler and turbine demands $[4,5]$. Tackling on the slow dynamic of combustion process, particularly in coal-fired power plants, is the greatest challenge in output pressure control, where linear or nonlinear mapping functions are employed in feedforward path to deal with this problem. The major problem of this method is that the essential parameters such as time delay should be exactly defined for model-based controllers; accordingly unless controllers retuning, any mismatches between the parameters of system may cause that the overall system becomes unstable [4]. In addition, due to physical limitation, using polynomial nonlinear maps may lead to unwanted oscillation by changing the operating conditions. Using nonlinear approximation functions such as neuro-fuzzy-based Hammerstein models can be an appropriate alternative. Hammerstein model is a block-oriented nonlinear model, which is able to incorporate the different dynamical behaviors of the system [18]. Many successful applications of fuzzy Hammerstein (FHS) have been reported for modeling the nonlinear behaviors of industrial processes $[18,19]$.

This paper focuses on the utilization of feedbackfeedforward coordinated control concept for improving the performance and maneuverability of a once-through power plant during load swings. In the proposed control system, neuro-fuzzy-based Hammerstein models are employed as the core of feedforward controller to generate the setpoint trajectories for the fuel, steam pressure and electrical power.
Input-output experimental data taken from real system responses over entire operating ranges is the basis for training the models. A genetic algorithm- (GA-) based fuzzy c-means (FCM) clustering method is employed for partitioning the entire operation range to smaller subregions and defining the structure of fuzzy models. By capturing the optimum parameters for membership functions, a least squares estimation technique is used to find the parameters of consequents with respect to steady-state data. Then, the parameters of the linear part can be obtained using a recursive least squares algorithm to fit the model responses on transient data. The developed models are used as the trajectory planner for the unit's subsystems. To diminish the effect of disturbances and to compensate deviations between variables and corresponding feedforward setpoints, feedback loops are considered. The performance of proposed control system is compared with the conventional turbine-follower control system installed on the real plant and also a modern turbine-follower coordinated control system in order to show the effectiveness and feasibility of the proposed control system.

So far, there are no reports on application of fuzzy Hammerstein models for trajectory planning in thermal power plants. In this work, the performance of the proposed approach has been evaluated for increasing the maneuverability of power plants during load changes.

This paper is organized as follows. In the next section, a general explanation of coordinated control structure is presented. The structure of fuzzy Hammerstein models is presented in Section 3. In Section 4, the training algorithms for identification of the linear and nonlinear parts of fuzzy Hammerstein are presented. In Section 5, the structure of feedback controllers and in Section 6, simulation experiments and control system design are presented. The control system tuning and comparison between the performances of proposed control system and the performances of other control systems are presented in Section 7. Finally, conclusion and some comments are provided in Section 8.

\section{Unit Load Control Scheme}

The concept of coordinated control is originated to keep the turbine and boiler operations together by providing a common demand signal. Removing any feedback from generated power on the boiler demands is a new idea adopted in new generation of coordinated control system. It is performed to prevent the rapid fluctuations in calorific value of the blast-furnace gas in the course of emerging electrical output disturbances [4]. The structure of coordinated control system is presented in Figure 1, where the dominant behavior of unit is governed by actuating elements (turbine valves and fuel supply) influenced simultaneously by load and pressure. The turbine master control regulates the turbine inlet pressure with respect to a predetermined value, where the electrical output is fed back on control valves. This is while the boiler control matching the boiler output pressure with the turbine demand. The setpoint signals for load demand and boiler fuel are trimmed by error signal defined as the difference between steam pressure at the boiler header and 


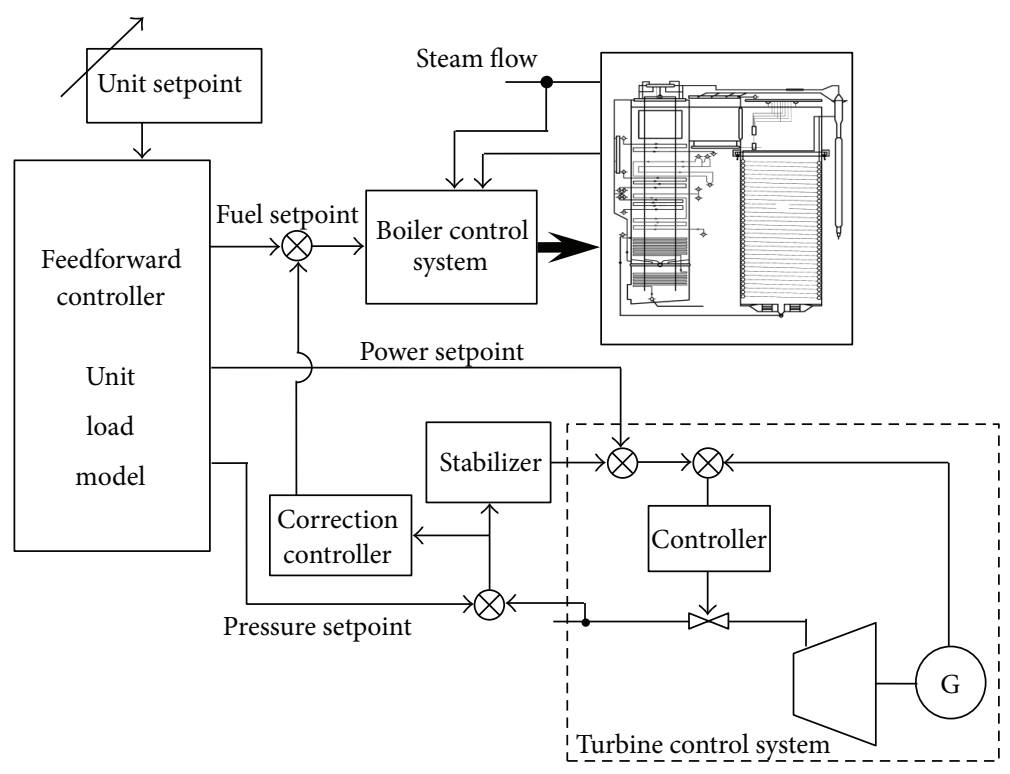

FIGURE 1: Coordinated control system configuration.

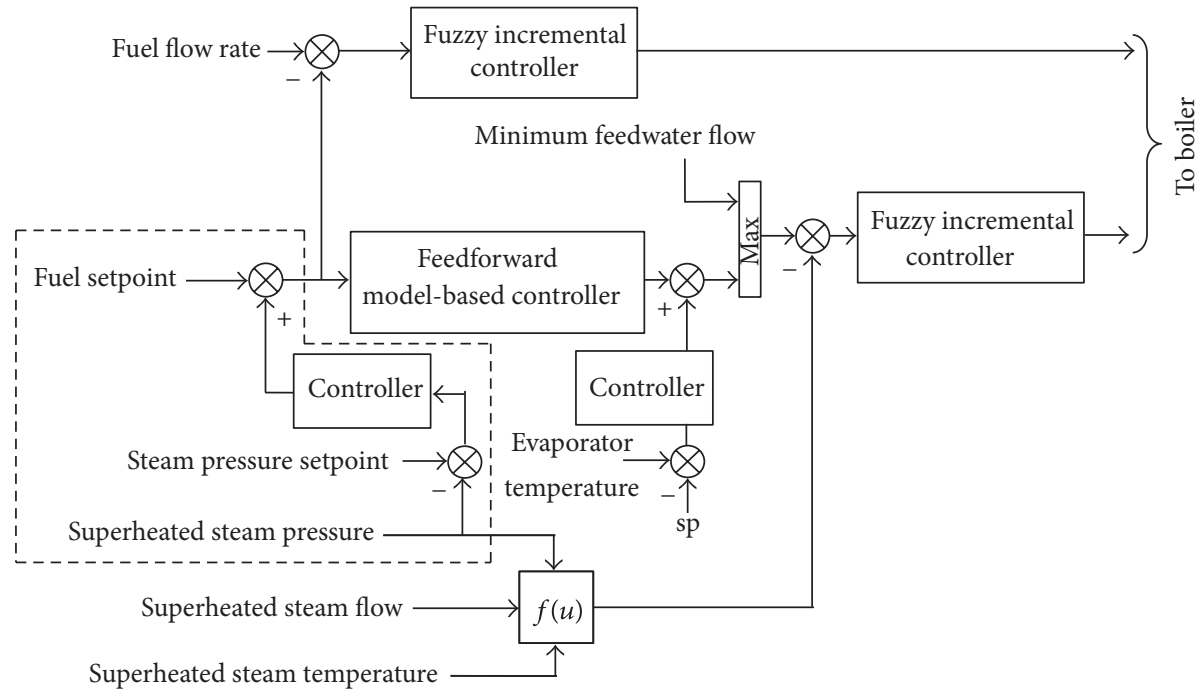

FIGURE 2: Boiler control system (fuel and feedwater).

its corresponding feedforward setpoint. This signal is also directly sent to the feedwater control system in order to compensate output pressure deviations. This configuration is completely decomposing the overall system to two separate subsystems $[20,21]$.

In addition, the boiler control system is presented in Figure 2. As it is shown, the correction controller compensates the fuel setpoint for the boiler. The required volume of feedwater is defined with respect to fuel flow references by using a feedforward model-based controller. The error signal from the evaporated steam temperature is also used as a correction signal in order to generate the steam flow rate reference.

A $440 \mathrm{MW}$ power generating unit, with a subcritical oncethrough Benson type boiler, is considered in this study. The nominal steam mass rate of the boiler is about $390 \mathrm{~kg} / \mathrm{s}$ (1405 ton/h), where the main superheated steam temperature is $535^{\circ} \mathrm{C}$ and the output steam pressure at full-load conditions is $18.6 \mathrm{MPa}$. In the next section, the proposed coordinated control system would be designed and applied to the accurate nonlinear models of this unit. The performances of the overall unit would be investigated as a new control system applied.

\section{The Structure of Fuzzy Hammerstein Model}

Improving the stability and performance of entire unit as well as smooth operation during load swings can be achieved through the reference trajectory planning. It is expected that the feedforward controller determines setpoints for the 


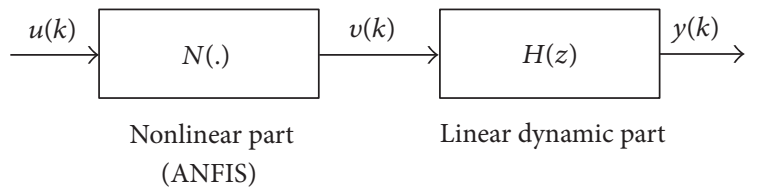

Figure 3: The structure of neuro-fuzzy-based Hammerstein model.

fuel, steam pressure, and electrical power with respect to the desired unit load defined by operator or dispatcher. In this paper, a fuzzy Hammerstein structure is employed to develop the unit load models, which are used as the core of feedforward controllers. The structure of fuzzy Hammerstein (FHS) model is presented in Figure 3. A Hammerstein model consists of a linear function, describing the system dynamics, and a nonlinear static function, $N(\cdot)$, describing the steadystate relations, as given by

$$
\begin{gathered}
y(k)=\sum_{i=1}^{n_{a}} a_{i} y(k-i)+\sum_{i=1}^{n_{b}} b_{i} v(k-i), \\
v(k)=N(u(k)),
\end{gathered}
$$

where $u(k)$ and $y(k)$ represent the variables of process input and output at the $k$ th sampling time, respectively, and $v(k)$ is the corresponding unmeasurable internal variable. In addition, $a_{i}\left(i=1,2, \ldots, n_{a}\right)$ and $b_{i}\left(i=1,2, \ldots, n_{b}\right)$ are the parameters of the model, where $n_{a}$ and $n_{b}$ are integers related to the model order [18]. The parameters $a$ and $b$ define a linear time-invariant dynamic system represented by the following equations:

$$
\begin{gathered}
y(t)=\frac{B\left(z^{-1}\right)}{A\left(z^{-1}\right)} v(t)+e(t), \\
A\left(z^{-1}\right)=1+a_{1} z^{-1}+\cdots+a_{n a} z^{-n a}, \\
B\left(z^{-1}\right)=1+b_{1} z^{-1}+\cdots+b_{n b} z^{-n b} .
\end{gathered}
$$

It is noted that developing accurate steady-state models for the nonlinear element of a Hammerstein model can be fairly difficult. Due to physical limitations, using polynomial nonlinear maps may lead to unwanted oscillation. It is suggested that nonlinear approximation functions such as neural networks, fuzzy model, or a combination of the approaches (neuro-fuzzy) can be used in order to overcome this problem [22].

The nonlinearity of the process $N(\cdot)$ was presented by the zero-order TSK (Takagi-Sugeno-Kang) type of fuzzy models [23]. A zero-order TSK fuzzy model can be expressed by a set of typical if-then rules as follows:

$$
R_{i}: \text { IF } u_{1} \text { is } \mu_{i, 1}, \ldots, u_{j} \text { is } \mu_{i, j} \text { THEN } v_{i}=\psi_{i} \text {, }
$$

where $R_{i}$ is the $i$ th fuzzy rule and $\mu_{i, j}$ is a membership function associated with input variable $u_{j}$. Also, $\psi_{i}$ is the zero order polynomial coefficient. Here, $j$ and $i$ are the number indices for the inputs and MFs, respectively. The fulfillment degrees of fuzzy rules are calculated through a five-layer feedforward network structure [23]. The weighted sum average according to fuzzy rules $R_{i}$ is obtained as follows:

$$
v=\sum_{i=1}^{N_{c}} v_{i} \bar{w}_{i}
$$

where

$$
\bar{w}_{i}=\frac{\prod_{j=1}^{N} \mu_{i, j}(\cdot)}{\sum_{i=1}^{N_{c}}\left[\prod_{j=1}^{N} \mu_{i, j}(\cdot)\right]},
$$

in which $N$ is the number of inputs to the fuzzy system and $N_{c}$ is the number of fuzzy rules. The membership function $\mu_{i, j}$ is considered to be Gaussian specified by the center $\sigma$ and the spread $\zeta$,

$$
\mu_{i, j}\left(u_{r}\right)=\exp \left(-\left(\frac{\left(u_{r}-\zeta_{i, j}\right)}{\sigma_{i, j}}\right)^{2}\right) .
$$

Therefore, with respect to (1) to (7), the fuzzy Hammerstein model can be presented as follows [24]:

$$
y(k)=\sum_{i=1}^{n_{a}} a_{i} y(k-i)+\sum_{i=1}^{n_{b}} b_{i} \sum_{j=1}^{N_{c}} \psi_{j}^{k-i} \bar{w}_{j},
$$

or

$$
y(k)=\sum_{i=1}^{n_{a}} a_{i} y(k-i)+\sum_{i=1}^{n_{b}} \sum_{j=1}^{N_{c}} b_{i} \psi_{j}^{k-i} \bar{w}_{j} .
$$

In (8), $a_{i}$ and $b_{i}$ are known as the linear parameters, where $\psi_{i}$ is nonlinear parameter. If the gain of the linear part is considered to be equal to one, the steady-state behavior can be described by the nonlinear part [24]. The structure of the fuzzy Hammerstein model is presented in Figure 4. By considering $b_{i}^{j}=b_{j} \psi_{i}$, we have

$$
\frac{b_{i}^{k}}{b_{j}^{k}} \frac{b_{j}^{l}}{b_{i}^{l}}=1 \quad \forall i, j, k, l \quad \text { or } \quad \frac{\psi_{i}}{\psi_{j}} \frac{\psi_{j}}{\psi_{l}}=1 \quad \forall i, j, k, l,
$$

which can transform the optimization problem to a constrained linear least squares estimation $[24,25]$.

In order to meet the requirement of generating stable and flat references trajectories, some assumptions are necessary to be considered. The function $N(\cdot)$ should be nonidentically null and bounded output for any bounded input (BIBO),

$$
\begin{gathered}
N(0)=0, \\
|N(u)|<\infty, \quad \text { for any inputs. }
\end{gathered}
$$

The most important required assumption is that the linear part of the model be stable,

$$
\forall \omega \in \mathbb{R}: z=e^{-j T \omega}, \quad\left|z^{n a} A\left(z^{-1}\right)\right|<1,
$$




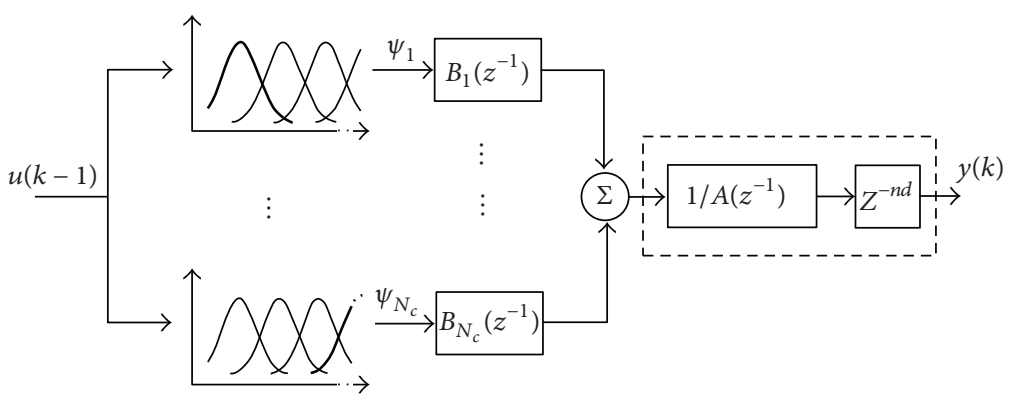

FIGURE 4: Linear and nonlinear parts plus time delay in a SISO fuzzy Hammerstein model.

where $T$ is the sampling time in discrete system. Including a delay in the real plant data can arise some difficulties by entering the time delay parameter in the model, which may cause unstable zeros and nonminimum phase dynamics to be appeared. In order to avoid such conditions, the time delay should be extracted from the model; therefore, the model becomes linear for the other parameters (Figure 4). In this case, a preprocessing is required to be performed on training data to remove the time delays.

It is also necessary for $A\left(z^{-1}\right)$ and $B\left(z^{-1}\right)$ to be coprime, which is guaranteed controllability of transfer function $B\left(z^{-1}\right) / A\left(z^{-1}\right)$. Furthermore, in order to prevent nonminimum phase behavior and have flat reference trajectories for fuel and generated power, the following condition is necessary:

$$
\forall \omega \in \mathbb{R}: z=e^{-j T \omega}, \quad\left|z^{n b} B\left(z^{-1}\right)\right|<1,
$$

which states that $B\left(z^{-1}\right)$ should have no unstable zero.

\section{The Training Algorithms}

In many cases, the linear and non-linear parts of models should be trained simultaneously. The major problem comes from the fact that the internal sequence $v(t)$ is not measurable. Due to independency of nonlinear static part $N(\cdot)$ and linear dynamic element $H(z)$, if the nonlinearity of system is known, then the training process can be performed in two separated stages.

4.1. Identification of Nonlinear Part. Despite the structure of the linear part, the model's nonlinear part can be identified based on steady-state data [18]. The training procedure of nonlinear part of model consists of two phases. First, in order to capture the structure of fuzzy part and to adjust the parameters of the membership functions, a genetic algorithm- (GA-) based fuzzy c-means (FCM) clustering method was employed. The FCM was originally presented by Dunn [26], in which the input data space is partitioning into $c$ predefined subsets through optimizing an objective function [27]. With respect to the similarity/dissimilarity of each cluster members, the dataset partitioning into clusters is preformed $[27,28]$.

An unlabeled dataset $X=\left\{x_{1}, \ldots, x_{n}\right\} \subset R^{s}$ can be partitioned into the given $c$ subsets represented as fuzzy sets $F=\left\{F_{1}, \ldots, F_{c}\right\}$ with cluster centroids vector $Z=$ $\left\{\zeta_{1}, \ldots, \zeta_{c}\right\} \subset R^{s}$. The similarity/dissimilarity of each cluster members is generally defined by the distance of data points from cluster centers. By defining the distance function $D\left(\zeta^{i}, x^{j}\right)$ as the Euclidean norm between $x^{i}$ and $\zeta^{j}$, we have

$$
D_{i j}^{2}=\left\|x_{j}-\zeta_{i}\right\|^{2}=\left(x_{j}-\zeta_{i}\right)^{T}\left(x_{j}-\zeta_{i}\right) .
$$

To find the best possible solution, the following c-means objective function has to be minimized:

$$
\operatorname{minimize}: J_{m}(\mathrm{Q}, \mathrm{Z})=\sum_{j=1}^{n} \sum_{i=1}^{c}\left(q_{i j}\right)^{m} D_{i j}^{2}
$$

where $Q=\left[q_{i j}\right]_{c \times n}$ and $q_{i j}$ are the membership degree of the $j$ th data point in the $i$ th cluster. The weighting exponent $m(1 \leq m<\infty)$ determines the degree of fuzziness for each datum membership. The membership matrix can be calculated by the following equation:

$$
q_{i j}=\left(\sum_{k=1}^{c}\left(\frac{D_{i j}}{D_{j k}}\right)^{2 /(m-1)}\right)^{-1}, \quad 1 \leq i \leq c, 1 \leq j \leq n .
$$

An optimization approach-based genetic algorithm (GA) was proposed by Chaibakhsh to solve the nonlinear optimization problem by minimizing the objective function $J_{m}$ [29]. The best possible cluster centers, $\left\{\zeta_{i}\right\}$, can be captured with respect to the following constraints on the membership values:

$$
\begin{gathered}
\forall j=1, \ldots, n, \quad \forall i=1, \ldots, c, \\
\sum_{i=1}^{c} q_{i j}=1, \quad 0 \leq q_{i j} \leq 1 .
\end{gathered}
$$

GAs are capable to discover global optimum by searching through wider solution spaces. The resistant to be trapped in local optimum points and the robustness in objective function evaluation are the other advantages of GAs. In the developed codes, the optimization problem is solved with respect to constraints, in which a simple Picard iteration algorithm was replaced by GA. The default optimization parameters for GA are presented in Table 1. More details about the developed codes for GA-based fuzzy clustering are presented in [29]. 
TABLE 1: The optimization parameters for genetic algorithm.

\begin{tabular}{lc}
\hline Optimization parameters & Type/value \\
\hline Population size & 100 \\
Crossover & Single point \\
Crossover rate & 0.7 \\
Mutation & Gaussian/scale $=1 /$ shrink $=0.7$ \\
Reproduction & Elite count: 2 \\
Selecting & Stochastic uniform \\
Migration & Forward $/ 0.1$ \\
Generations & 200 \\
\hline
\end{tabular}

The whole operating range can be divided to smaller regimes using clustering method. It is noted that the number of fuzzy rules is equal to the number of cluster centers. Thus, it is necessary to define the number of cluster centers to achieve an acceptable degree of accuracy. It is recommended that the number of cluster centers is chosen to be four to divide the operating range into four subregions [29].

By defining the structure of the consequent, in the second stage, the parameters of fuzzy rules can be adjusted using least squares estimation (LSE) technique. By considering $\widehat{\Psi}=$ $\left[\begin{array}{llll}\psi_{1} & \psi_{2} & \cdots & \psi_{N_{c}}\end{array}\right]^{T}$ as the estimated model parameters and

$$
\begin{gathered}
Y=\left[\begin{array}{cccc}
y(1) & y(2) & \cdots & y(n)
\end{array}\right]_{n \times 1}^{T}, \\
X=\left[\begin{array}{ll}
\bar{w}_{i}^{j} & { }_{n \times N_{c}},
\end{array}\right.
\end{gathered}
$$

with respect to (11), by some simplifications, the linear equality constraints can be considered as

$$
Q \Psi=0
$$

where

$$
Q=\left[\begin{array}{cccccc}
1 & 0 & -1 & 0 & \cdots & 0 \\
0 & 1 & 0 & -1 & \cdots & 0 \\
\cdot & \cdot & \cdot & \cdot & \cdot & \cdot \\
\cdot & \cdot & \cdot & \cdot & \cdot & \cdot \\
\cdot & \cdot & \cdot & \cdot & \cdot & \cdot \\
0 & \cdots & 0 & 1 & 0 & -1
\end{array}\right]
$$

Therefore, the constrained least squares (CLSs) estimation problem can be captured by as follows [30]:

$$
\begin{gathered}
\widehat{\Psi}=\left(X^{T} X\right)^{-1} X^{T} Y, \\
\widehat{\Psi}^{\mathcal{C}}=\widehat{\Psi}-\left(X^{T} X\right)^{-1} Q^{T}\left(Q\left(X^{T} X\right)^{-1} Q^{T}\right)^{-1} Q \widehat{\Psi} .
\end{gathered}
$$

4.2. Identification of Linear Part. By adjusting the consequents' parameters of fuzzy part, the parameters of linear part can be tuned based on transient data taken from a real system. With respect to Figure 4, the linear part of fuzzy Hammerstein models can be considered as a linear multi-input multioutput (MISO) system.
It is noted that the gain of the linear part has to be normalized and be equal to one [24].

$$
\frac{\sum_{1}^{n_{b}} b_{i}}{\left(1-\sum_{1}^{n_{a}} a_{i}\right)}=1 \text {. }
$$

Therefore, we define the regressor vector $\Phi$ as follows:

$$
\begin{aligned}
\Phi_{k-1}= & {\left[y(k-1), y(k-2), \ldots, y\left(k-n_{a}\right),\right.} \\
& \left.\sum_{j=1}^{N_{c}} \psi_{j}^{k-1} \bar{w}_{j}^{k-1}, \sum_{j=1}^{N_{c}} \psi_{j}^{k-2} \bar{w}_{j}^{k-2}, \ldots, \sum_{j=1}^{N_{c}} \psi_{j}^{k-n_{b}} \bar{w}_{j}^{k-n_{b}}\right],
\end{aligned}
$$

where $N_{c}$ is equal to $c$ (the number of cluster centers). Also, the parameter vector $\Theta$ is as,

$$
\Theta_{k}=\left[\begin{array}{llllllll}
\hat{a}_{1} & \hat{a}_{2} & \cdots & \hat{a}_{n a} & \hat{b}_{1} & \hat{b}_{2} & \cdots & \hat{b}_{n b}
\end{array}\right] .
$$

The parameter vector $\Theta$ can be estimated by recursive least squares algorithm with the exponential forgetting factor $\lambda$ as follows:

$$
\begin{gathered}
\widehat{\Theta}_{k}=\widehat{\Theta}_{k-1}+K_{k} \mathrm{E}_{k}, \\
\mathrm{E}_{k}=\left(Y_{d}-\Phi_{k}^{T} \widehat{\Theta}_{k-1}\right), \\
K_{k}=\left(\lambda+\Phi_{k}^{T} P_{k-1} \Phi_{k}\right)^{-1} P_{k-1} \Phi_{k}, \\
P_{k}=\lambda^{-1}\left(I-K_{k} \Phi_{k}^{T}\right) P_{k-1},
\end{gathered}
$$

in which $P$ and $K$ are the covariance matrix and (so called) the Kalman gain matrix, respectively. Also, the forgetting factor $\lambda$ is considered to be 0.9 . By choosing a well-distributed sequence of data over the desired range, the linear part of fuzzy Hammerstein model can be obtained.

\section{Feedback Controllers}

In fact, there is no mathematical model that can exactly describe unit load dynamics, and therefore, there is always a mismatching (uncertainty) between generated setpoints by feedforward controller and system variables. Besides, serving disturbances from unknown sources is almost expected in such systems (fuel quality, electrical disturbances, etc.). In this case, feedback control is required to compensate the uncertainty and eliminate disturbances in order to track the unit demand. As it is shown in Figure 1, the difference between the pressure reference and the measured output pressure signals is used to adjust the fuel and power references through feedback path. Here, two types of fuzzy logic controllers (FLCs) are employed as the feedback controllers.

First type is a PD-FLC controller, in which the error $(e)$ and rate of error changes $(\dot{e})$ are considered as its inputs (Figure 5(a)). The input membership functions are chosen to be Gaussian, which are symmetrically distributed with cardinality of five over interval range $[-1,1]$. The output 
TABLE 2: Fuzzy rules for fuzzy controller.

\begin{tabular}{lccccc}
\hline$e(k)$ & & \multicolumn{4}{c}{$\Delta e(k)$} \\
& NL & NS & ZE & PS & PL \\
\hline NL & NL & NM & NM & NS & NS \\
NS & NM & NS & NS & ZE & ZE \\
ZE & NS & NS & ZE & PS & PS \\
PS & ZE & ZE & PS & PS & PM \\
PL & PS & PS & PM & PM & PL \\
\hline
\end{tabular}

membership functions in triangular form are extended with cardinality of seven over the interval range $[-1,1]$. A combination of 25 if-then rules is required for correction controllers, which is presented in Table 2 . The fuzzy inference method employs the minimum function as $T$-norm and the centroid method with min-max operator as the defuzzification strategy.

The second type of employed FLC is an incremental algorithm considered for boiler controllers, such that only small corrections are added to controllers output. As shown in Figure 5(b), the incremental controller generates the future command signal by adding a change in control signal $\Delta u$ to the current control signal $u$,

$$
u_{n}=u_{n-1}+\Delta u_{n}
$$

The input scaling factors, which transform crisps inputs into a normalized universe, are chosen with respect to the maximum value of error and error changes. The output gains are tuned by performing some simulation tests in order to achieve the best possible results.

\section{Simulation Experiments and Control System Design}

In this section, the fuzzy Hammerstein models are developed based on experimental data from real plant performances. In addition, by performing some simulation experiments, the developed model for the pressure reference is retuned to meet the plant's new conditions. Finally, the unit models are employed as the core of feedforward controllers.

In order to perform simulation experiments, a detailed nonlinear model of a $440 \mathrm{MW}$ once-through power plant unit is employed. This model comprises smaller components that are developed based on the thermodynamics principles and energy balance. The parameters of developed models are determined either from constructional data or by applying genetic algorithm (GA) techniques on the experimental data. The modeling approach and the models parameters optimization technique for the turbine and boiler subsections are extensively presented in $[31,32]$. The proposed control system is applied to this model in MATLAB SIMULINK environment. Simulations are run when the same inputs as the actual system are applied.

The actual data used for modeling and simulation purposes are taken by a complete set of field experiments, as the unit load changes between 45 to 100 percent of the nominal load (195 440 MW). The data are recorded as discrete
TABLE 3: The error functions for the unit's models.

\begin{tabular}{lccccc}
\hline Model & Max $(|e|)$ & Min $(|e|)$ & Mean & MAE & $R^{2}$ \\
\hline Load & 16.1539 & 0.0012 & -0.1483 & 1.0406 & 0.9967 \\
Fuel & 1.2777 & $1.43 e-5$ & -0.0187 & 0.0857 & 0.9989 \\
Feedwater & 10.4450 & $3.0116 e-4$ & 0.2048 & 0.9901 & 0.9976 \\
Pressure & 0.3611 & $7.5 e-7$ & 0.02 & 0.0632 & 0.9994 \\
\hline
\end{tabular}

time signals with five seconds sampling time, consisting of transient and steady-state conditions (45 65, 65 75, 75 90, and 90 100 percent). By applying the proposed modeling approach to the steady-state and transient data, the linear and non-linear part of fuzzy Hammerstein models can be developed. The accuracy of the developed models is assessed by comparing the responses of the models and the real plant performances. For this aim, the error functions are calculated by defining the error as the difference between the predicted values by the models and the experimental data. Here, the upper bound of error (Max $(|e|))$, lower bound of error (Min $(|e|))$, mean absolute error (MAE), and the coefficient of determination $\left(R^{2}\right)$ are calculated by the following equations:

$$
\begin{gathered}
\text { Mean }=\frac{1}{n} \sum_{i=1}^{n}\left(y_{i}^{*}-y_{p}^{(i)}\right), \\
\text { MAE }=\frac{1}{n} \sum_{i=1}^{n}\left|y_{i}^{*}-y_{p}^{(i)}\right|, \\
R^{2}=1-\frac{\sum_{i=1}^{n}\left(y_{i}^{*}-y_{p}^{(i)}\right)^{2}}{\sum_{i=1}^{n}\left(y_{i}^{*}-\bar{y}\right)^{2}},
\end{gathered}
$$

where $\bar{y}$ is the average of $y$ over the $n$ samples and $y_{i}^{*}$ and $y_{p}^{(i)}$ are the $i$ th target and the model output, respectively. The error functions for the developed models are presented in Table 3.

The load reference model can be captured based on experimental data as a linear single-input single-output model. However, the load trajectory can be modified to meet the new unit's requirements to increase the overall performances of the plant. Therefore, the following transfer function is proposed for the load trajectory planner:

$$
G_{\text {load }}(z)=z^{-40} \times \frac{10^{-4}(2.7319 z+2.68677)}{z^{2}-1.9507 z+0.9512},
$$

where the second-order model comprises one stable zero and 40 sequences delay. The responses of the developed model for load is presented in Figure 6(a). For this model, the error functions MAE and $R^{2}$ are determined as a 1.0406 and 0.9967.

In Figure 6(b), the responses of the developed model for fuel are compared with real system responses in order to validate the accuracy and performance of the developed model.

In once-through boilers, the required quantity of feedwater relates to the fuel flow rate. However, this relation is not constant and would vary by load changes. In this case, the relation between fuel and feedwater flow rates can be predicted by employing an FHS model. The responses of the 


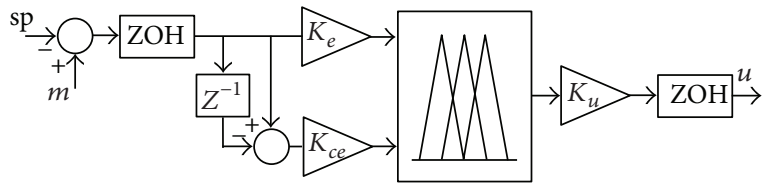

(a)

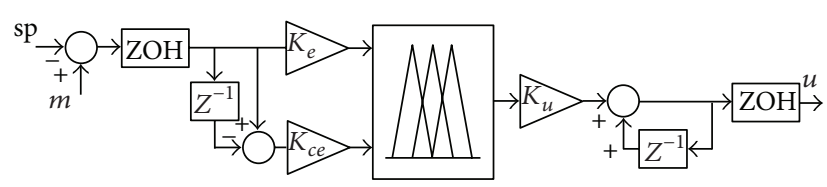

(b)

FIGURE 5: (a) PD-fuzzy logic controller and (b) incremental fuzzy logic controller.

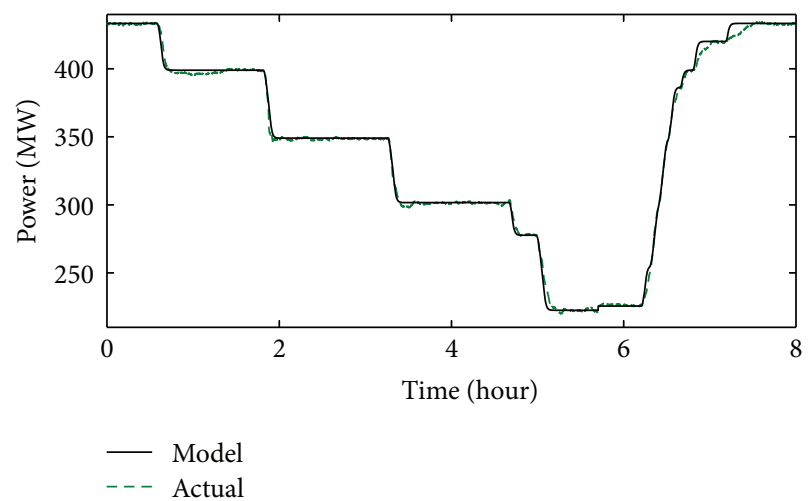

(a)

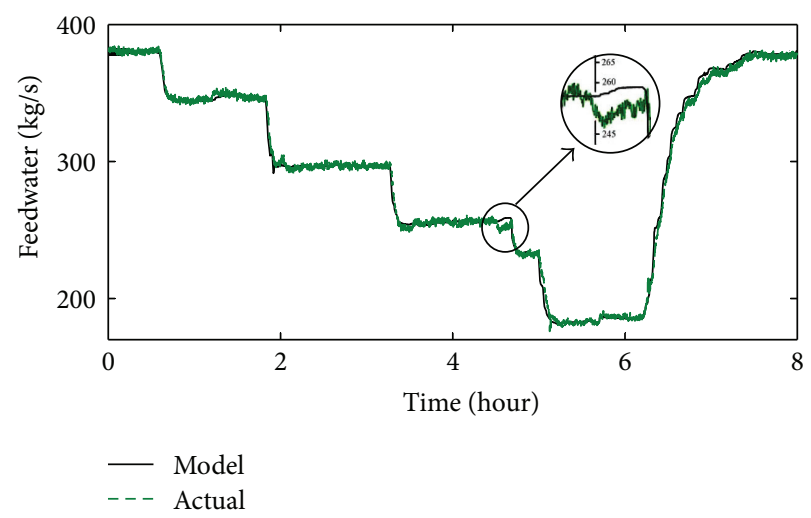

(c)

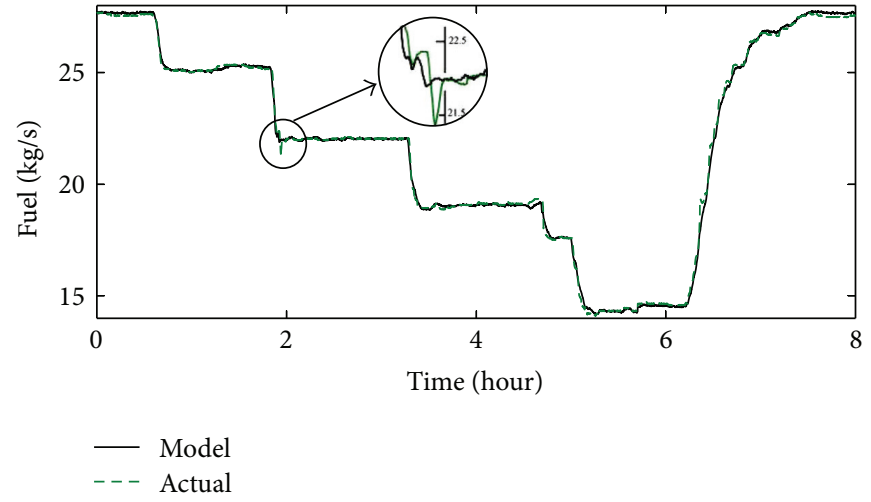

(b)

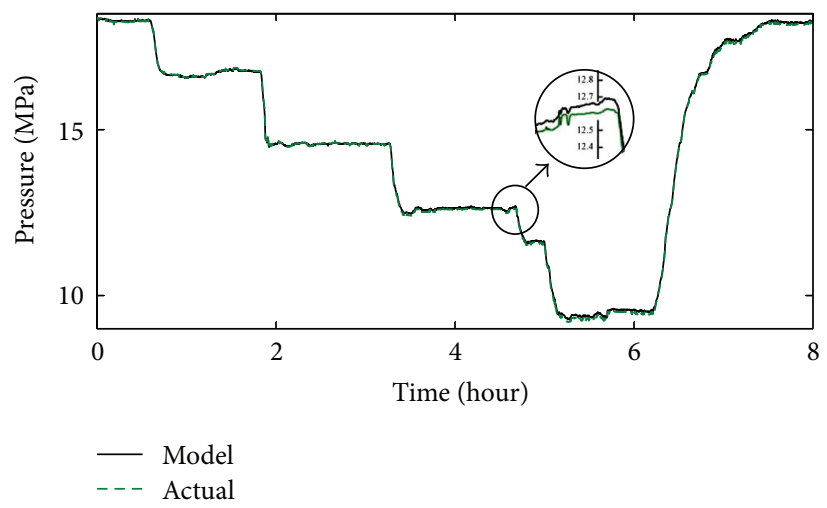

(d)

FIGURE 6: The responses of fuzzy Hammerstein models for the unit's subsystem.

developed model for the feedwater are presented in Figure $6(c)$. This model is used as the core of a feedforward controller in order to compensate the nonlinear behavior of the boiler and to derive the required volume of feedwater with respect to fuel flow (Figure 2). The error functions MAE and $R^{2}$ for the feedwater model are determined as 0.9901 and 0.9976 .

It should be noted that the setpoint for steam pressure plays the main role in coordinating the boiler and turbine operation, where very small tracking error may cause large oscillations in the system. By applying a new trend for load changes, the pressure references should be modified with respect to new operating conditions. However, the steadystate conditions of the unit are not changed, and therefore, the non-linear part of the model can be captured based on the recorded data. In order to obtain the linear part of the pressure model, a cyclic test was performed as the load swung between $50 \%$ to $100 \%$ load. For this propose, the correction controllers should temporary be disabled. The obtained data from simulation experiments are employed to tune the linear part of the pressure model. In Figure 6(d), the responses of the developed model for the pressure are shown. As it is presented in Table 3, the maximum deviations between the training data and predicted values are not more than 0.37. Also, the error functions MAE and $R^{2}$ are determined as 0.0632 and 0.9994 , which indicates the accuracy of the developed model for the pressure trajectory planner.

The developed FHS models are used as the reference governors for the generated power, fuel, steam pressure, and also feedwater flow rate. The reference signals for the fuel and generated power are trimmed with respect to boiler pressure deviations from the pressure reference by means of feedback controllers. 


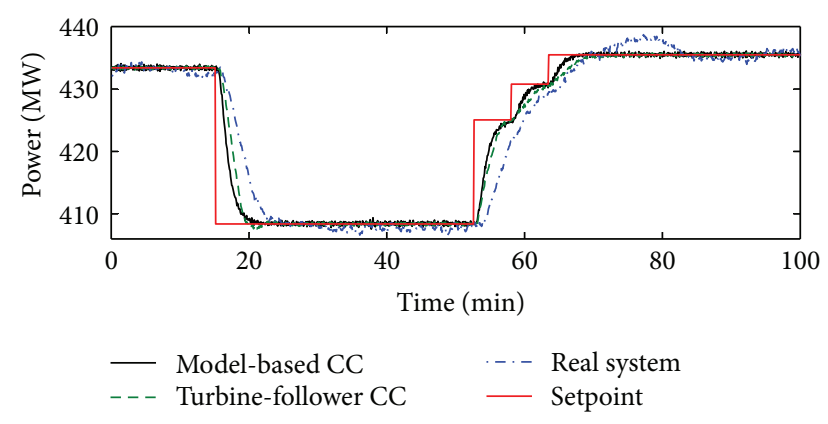

(a)

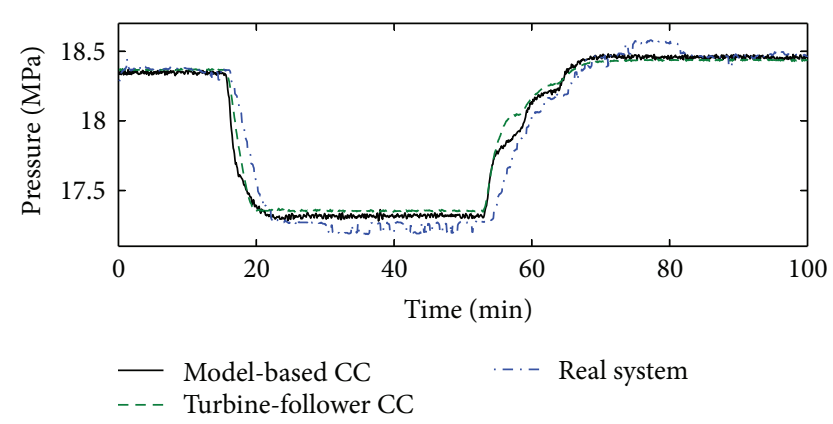

(b)

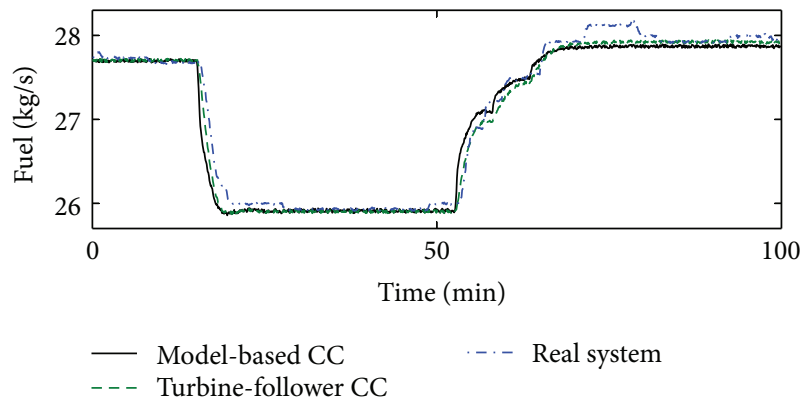

(c)

FIGURE 7: The unit responses at high-load conditions ((a) power, (b) pressure, and (c) fuel).

\section{Results and Discussion}

In this section, the performance of the designed coordinated control system is evaluated. A comparison was preformed between the responses of corresponding system and the real plant responses over a wide range of operations.

The real system utilizes a conventional turbine-follower control structure [33]. In this structure, the generated power is compared with the desired load signal, which is used to set the boiler demands. The boiler output pressure is adjusted by a mechanical governor. This technique is persistently suffering from the inherent time delay and the large inertia associated with combustion system. This may lead to produce inner disturbances by emerging changes in the quality of fuel. Furthermore, employing conventional PI- or PID-based control schemes in such system may cause that the control system fails to reject disturbances, noise, and uncertainties, due to aging effects. The performance of proposed control

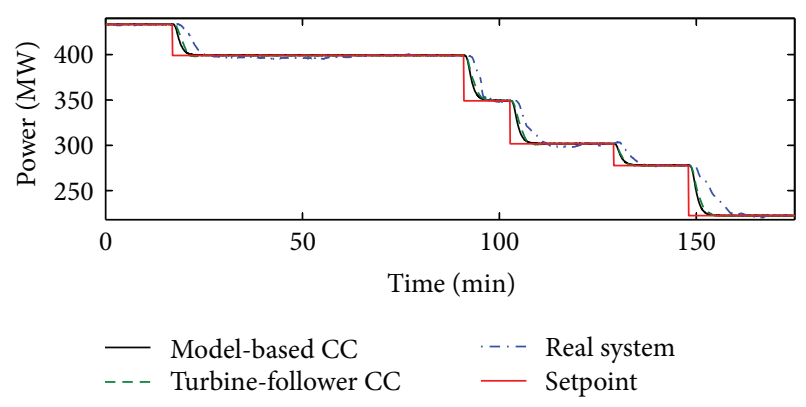

(a)

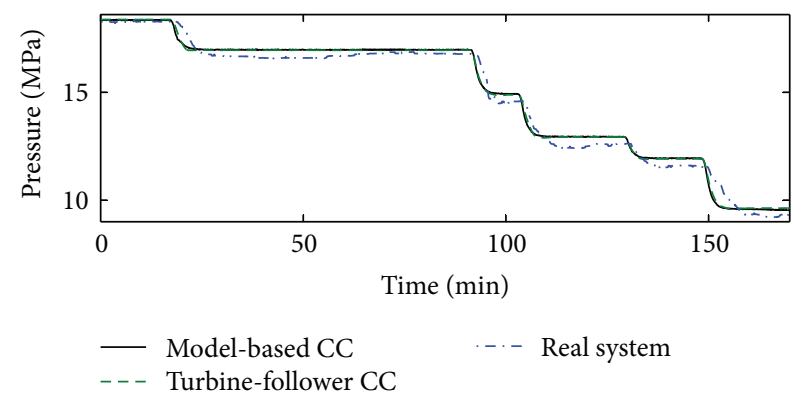

(b)

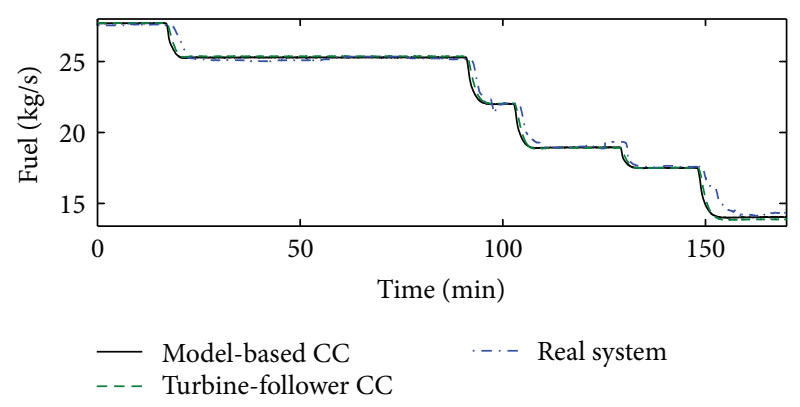

(c)

FIGURE 8: The unit responses as the load is ramped down from fullload to half-load conditions ((a) power, (b) pressure, and (c) fuel).

system is also compared with a turbine-follower coordinated (TFC) system $[33,34]$.

The responses of the unit, as the load is at high-load conditions and swings between 92 to 98 percent of nominal load, are presented in Figure 7(a). Obtained results indicate the faster responses of the unit and higher load tracking performance during load changes with the proposed modelbased CC. Also, the boiler steam pressure is presented in Figure 7(b). The steam pressure at boiler output header is stabilized as load changes by turbine-follower and modelbased control system, where more fluctuations in the steam pressure can be observed in the real system. The fuel flow rate is also shown in Figure 7(c). In the proposed control system, there is no feedback from generated load on the fuel, which allows the boiler to response with higher gradient rates.

The responses of the unit, as the load is ramped down from full-load to half-load conditions and also as it is ramped up from half-load to full-load conditions, are presented in Figures 8 and 9. With conventional control system, the 


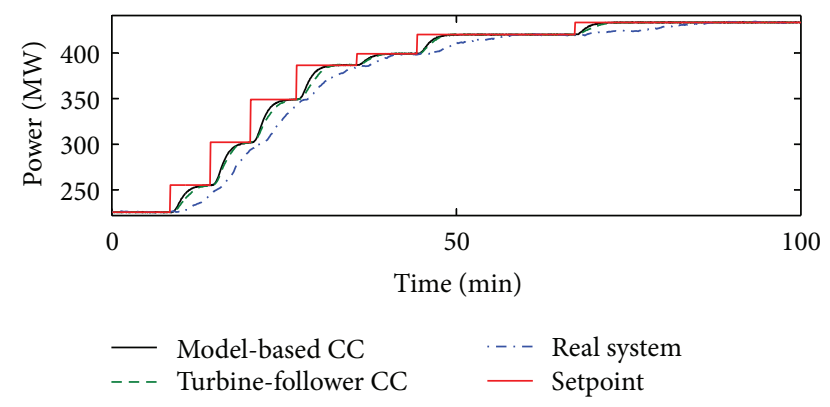

(a)

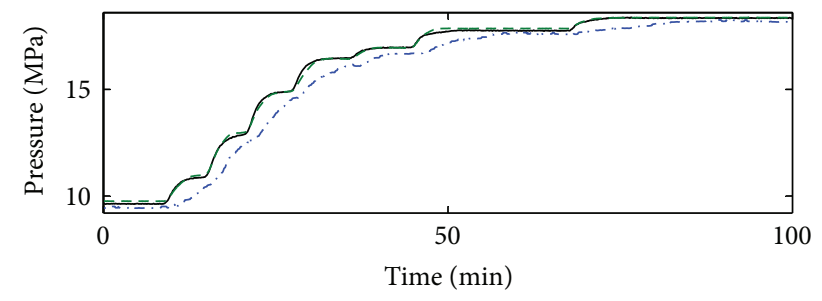

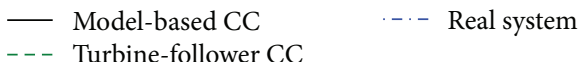

(b)

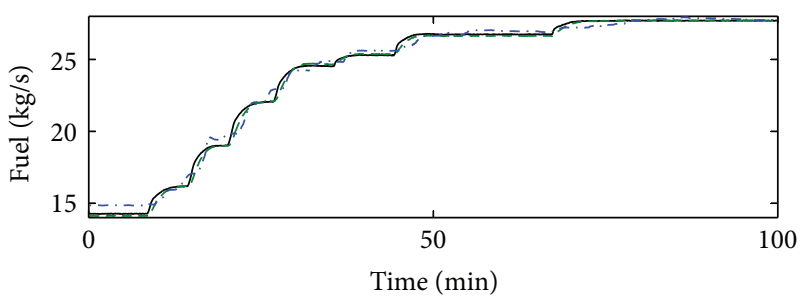

$\begin{array}{ll}\text { - } & \text { Model-based CC } \\ \text { - - } & \text { Turbine-follower CC }\end{array}$

(c)

FIGURE 9: The unit responses as the load is ramped up from half-load to full-load conditions ((a) power, (b) pressure, and (c) fuel).

load gradients are considered to be limited at $10 \% / \mathrm{min}$ and $2 \% / \mathrm{min}$ for medium-load and low/high-load ranges, respectively. As it is shown in Figures 8(a) and 9(a), by employing the proposed control system, the load gradients are increased up to $12 \% / \mathrm{min}$ and $6 \% / \mathrm{min}$ for mediumload and low/high-load conditions, respectively. In Figures 8 (b) and 9(b), the steam pressure responses are presented. Obtained results show that the steam pressure is perfectly stable with model-based and turbine-follower CC systems. Figures $8(\mathrm{c})$ and $9(\mathrm{c})$ are presenting the fuel flow rate at different operating conditions. Simulation results show that emerging any disturbances at the output electrical power has no effect on the combustion system. During sudden load changes, no considerable overfiring/underfiring is needed to meet the new load setting. This leads to smooth and stable operation of the entire unit.

\section{Conclusion}

In this paper, a nonlinear coordinated control concept is presented in order to improve the flexibility and the performance of a once-through power plant. The control system was consisting of a model-based feedforward controller, which generated the boiler demand and turbine references with respect to desired load. The core of feedforward controller was a neuro-fuzzy-based Hammerstein model, which were developed based on experimental data taken from real system responses. In order to eliminate effect of disturbances and compensate the deviations between the control variables and corresponding feedforward setpoints, fuzzy logic controllers were employed in feedback paths. The load demand signals to turbine and boiler were trimmed by error signals coming from correction units. The proposed coordinated control system has been designed and evaluated by using nonlinear boiler-turbine model through computer simulations.

The responses of plant with new coordinated control were compared with the real plant responses over a wide range of operations, where the real system has a conventional turbine-follower control strategy. Simulation results indicate the performance of proposed control system during load changes. Greater load tracking and stabilizing the steam pressure at the boiler output header are the remarkable capabilities of this control system.

In addition, the performance of the proposed control system was compared with the turbine-follower CC strategy. The results showed that the new model-based CC concept could significantly enhance the maneuverability and loadfollowing capability of the power plant over a wide range of operation.

\section{References}

[1] S. Daley, "Advances in power plant control and monitoring," Computing and Control Engineering Journal, vol. 12, no. 2, pp. 51-52, 2001.

[2] S. J. Qin and T. A. Badgwell, "A survey of industrial model predictive control technology," Control Engineering Practice, vol. 11, no. 7, pp. 733-764, 2003.

[3] A. C. F. Guimarães and C. M. F. Lapa, "Parametric fuzzy study for effects analysis of age on PWR containment cooling system," Applied Soft Computing Journal, vol. 8, no. 4, pp. 1562-1571, 2008.

[4] G. K. Lausterer, "Improved maneuverability of power plants for better grid stability," Control Engineering Practice, vol. 6, no. 12, pp. 1549-1557, 1998.

[5] R. Garduno-Ramirez and K. Y. Lee, "Wide range operation of a power unit via feedforward fuzzy control," IEEE Transactions on Energy Conversion, vol. 15, no. 4, pp. 421-426, 2000.

[6] C. K. Weng and A. Ray, "Robust wide-range control of steamelectric power plants," IEEE Transactions on Control Systems Technology, vol. 5, no. 1, pp. 74-88, 1997.

[7] T. Yu, K. W. Chan, J. P. Tong, B. Zhou, and D. H. Li, "Coordinated robust nonlinear boiler-turbine-generator control systems via approximate dynamic feedback linearization," Journal of Process Control, vol. 20, no. 4, pp. 365-374, 2010.

[8] Y. Daren and X. Zhiqiang, "Nonlinear coordinated control of drum boiler power unit based on feedback linearization," IEEE 
Transactions on Energy Conversion, vol. 20, no. 1, pp. 204-210, 2005.

[9] G. O. Guardabassi and S. M. Savaresi, "Approximate linearization via feedback - an overview," Automatica, vol. 37, no. 1, pp. $1-15,2001$.

[10] N. Yadaiah, A. Ganga Dinesh Kumar, and J. L. Bhattacharya, "Fuzzy based coordinated controller for power system stability and voltage regulation," Electric Power Systems Research, vol. 69, no. 2-3, pp. 169-177, 2004.

[11] E. J. Adam and J. L. Marchetti, "Designing and tuning robust feedforward controllers," Computers and Chemical Engineering, vol. 28, no. 9, pp. 1899-1911, 2004.

[12] C. M. Cirre, M. Berenguel, L. Valenzuela, and E. F. Camacho, "Feedback linearization control for a distributed solar collector field," Control Engineering Practice, vol. 15, no. 12, pp. 1533-1544, 2007.

[13] A. R. Martinez and R. G. Ramirez, "2 DOF fuzzy gainScheduling PI for combustion turbogenerator speed control," in Proceedings of the IFAC Conference on Advances in PID Control, Brescia, Italy, 2012.

[14] M. Santos and A. L. Dexter, "Control of a cryogenic process using a fuzzy PID scheduler," Control Engineering Practice, vol. 10, no. 10, pp. 1147-1152, 2002.

[15] F. Claveau, P. Chevrel, and K. Knittel, "A 2DOF gain-scheduled controller design methodology for a multi-motor web transport system," Control Engineering Practice, vol. 16, no. 5, pp. 609-622, 2008.

[16] P. Kallappa and A. Ray, "Fuzzy wide-range control of fossil power plants for life extension and robust performance," Automatica, vol. 36, no. 1, pp. 69-82, 2000.

[17] A. Piazzi and A. Visioli, "A noncausal approach for PID control," Journal of Process Control, vol. 16, no. 8, pp. 831-843, 2006.

[18] G. Harnischmacher and W. Marquardt, "A multi-variate Hammerstein model for processes with input directionality," Journal of Process Control, vol. 17, no. 6, pp. 539-550, 2007.

[19] L. Jia, M. S. Chiu, and S. S. Ge, "A noniterative neurofuzzy based identification method for Hammerstein processes," Journal of Process Control, vol. 15, no. 7, pp. 749-761, 2005.

[20] S. Li, H. Liu, W. J. Cai, Y. C. Soh, and L. H. Xie, "A new coordinated control strategy for boiler-turbine system of coal-fired power plant," IEEE Transactions on Control Systems Technology, vol. 13, no. 6, pp. 943-954, 2005.

[21] H. C. Gery, "Evolution of coordinated control," in Proceedings of the Instrument Society of American Power Symposium Instrumentation in the Power Industry, vol. 31, pp. 23-25, St. Petersburg, Russia, May 1988.

[22] L. Jia, M.-S. Chiu, and S. S. Ge, "Iterative identification of neurofuzzy-based Hammerstein model with global convergence," Industrial and Engineering Chemistry Research, vol. 44, no. 6, pp. 1823-1831, 2005.

[23] T. Takagi and M. Sugeno, "Fuzzy identification of systems and its applications to modeling and control," IEEE Transactions on Systems, Man and Cybernetics, vol. 15, no. 1, pp. 116-132, 1985.

[24] J. Abonyi, R. Babuška, M. Ayala Botto, F. Szeifert, and L. Nagy, "Identification and control of nonlinear systems using Fuzzy Hammerstein models," Industrial and Engineering Chemistry Research, vol. 39, no. 11, pp. 4302-4314, 2000.

[25] W. D. Timmons, H. J. Chizeck, F. Casas, V. Chankong, and P. G. Katona, "Parameter-constrained adaptive control," Industrial and Engineering Chemistry Research, vol. 36, no. 11, pp. 4894-4905, 1997.
[26] J. C. Dunn, "A fuzzy relative of the ISODATA process and its use in detecting compact well-separated clusters," Journal of Cybernetics, vol. 3, no. 3, pp. 32-57, 1973.

[27] J. C. Bezdek, R. Ehrlich, and W. Full, "FCM: the fuzzy c-means clustering algorithm," Computers and Geosciences, vol. 10, no. 2-3, pp. 191-203, 1984.

[28] N. R. Pal, K. Pal, J. M. Keller, and J. C. Bezdek, "A possibilistic fuzzy c-means clustering algorithm," IEEE Transactions on Fuzzy Systems, vol. 13, no. 4, pp. 517-530, 2005.

[29] A. Chaibakhsh, "Modelling and long-term simulation of a heat recovery steam generator," Mathematical and Computer Modelling of Dynamical Systems. IFirst, pp. 1-24, 2012.

[30] T. Amemiya, Advanced Econometrics, Harvard University Press, 1985.

[31] A. Chaibakhsh and A. Ghaffari, "Steam turbine model," Simulation Modelling Practice and Theory, vol. 16, no. 9, pp. 1145-1162, 2008.

[32] A. Chaibakhsh, A. Ghaffari, and S. A. A. Moosavian, "A simulated model for a once-through boiler by parameter adjustment based on genetic algorithms," Simulation Modelling Practice and Theory, vol. 15, no. 9, pp. 1029-1051, 2007.

[33] P. M. Anderson and A. A. Fouad, Power System Control and Stability, Wiley, New York, NY, USA, 2nd edition, 2003.

[34] F. P. de Mello, "Dynamic models for fossil fueled steam units in power system studies," IEEE Transactions on Power Systems, vol. 6, no. 2, pp. 753-761, 1991. 

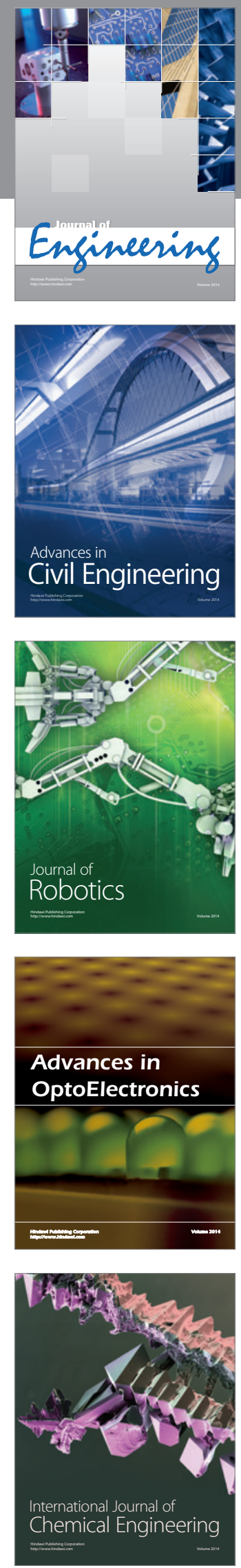

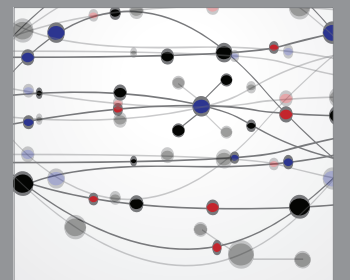

The Scientific World Journal
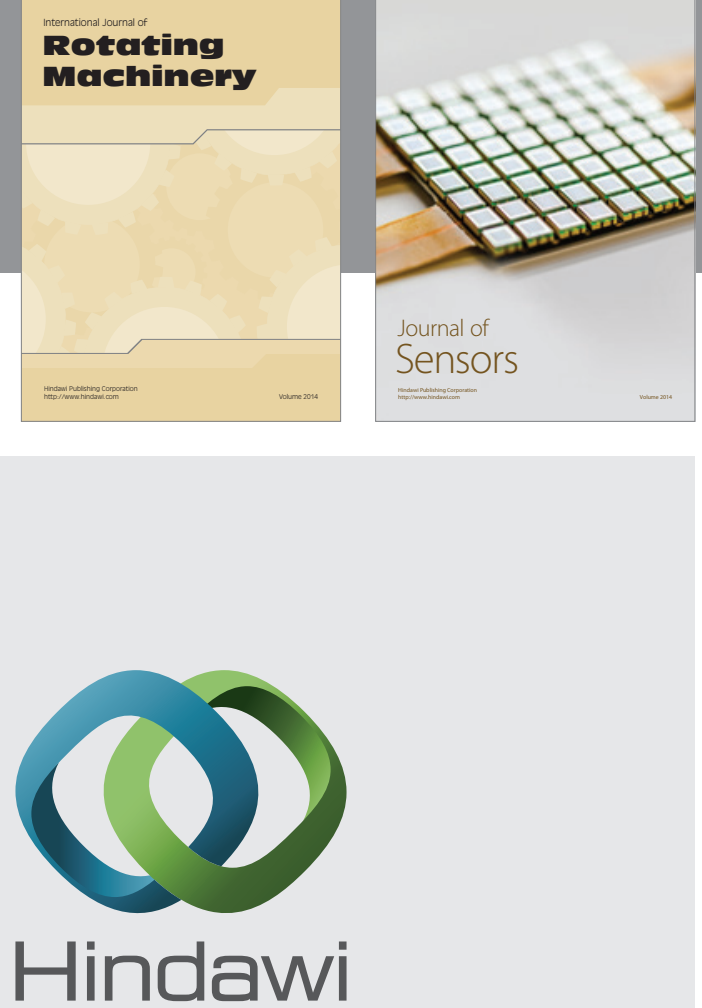

Submit your manuscripts at http://www.hindawi.com
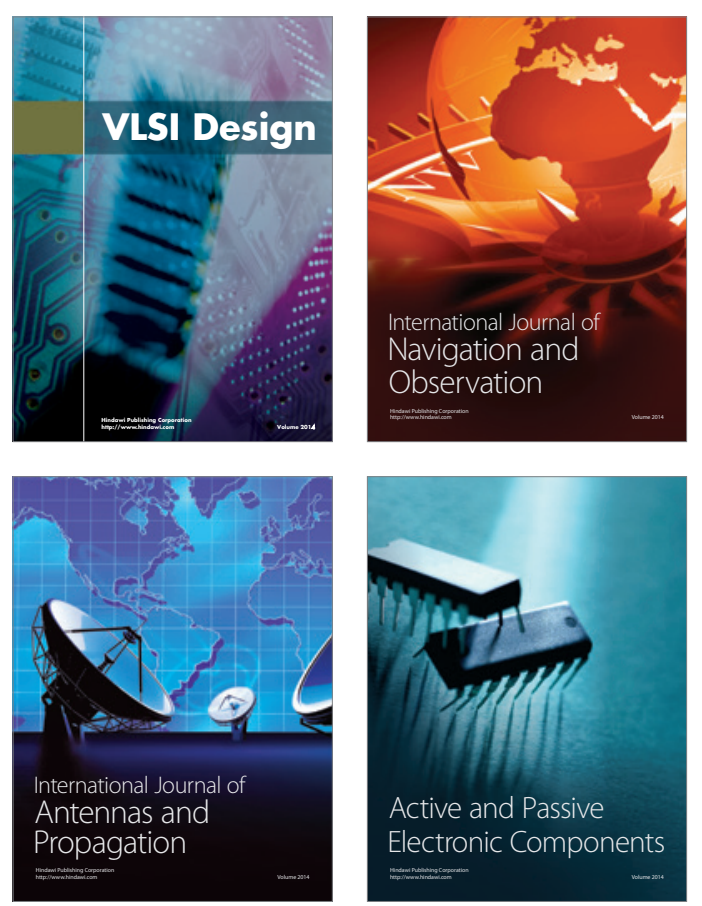
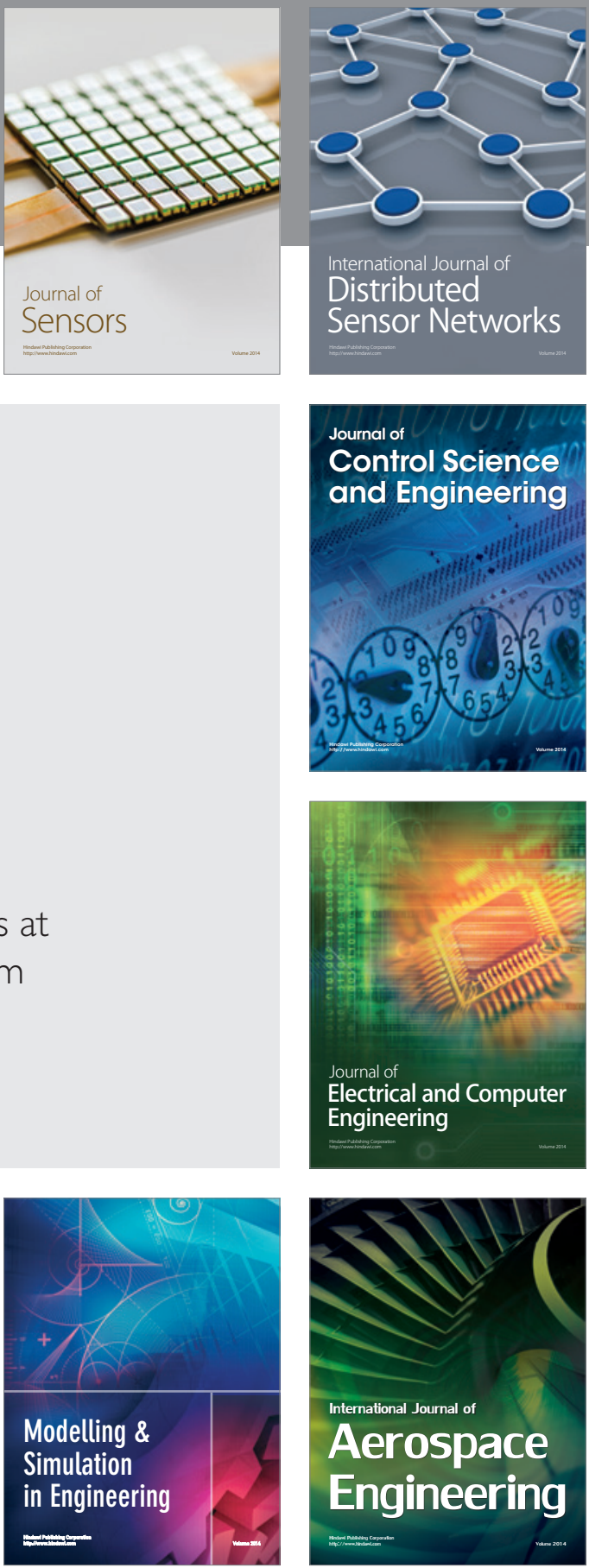

Journal of

Control Science

and Engineering
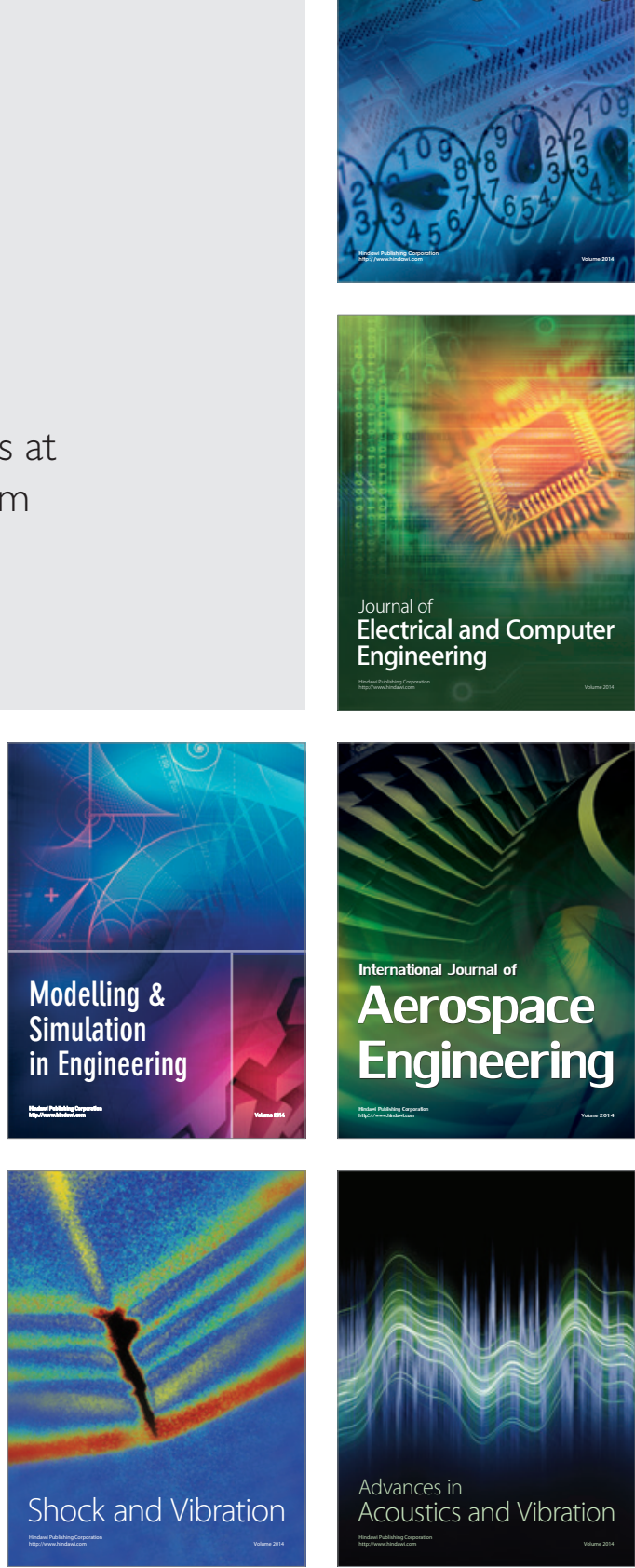